\title{
The clinical effects of low-dose splenic irradiation combined with chest three-dimensional conformal radiotherapy on patients with locally advanced non- small-cell lung cancer: a randomized clinical trial
}

\author{
This article was published in the following Dove Press journal: \\ OncoTargets and Therapy \\ 7 September 2016 \\ Number of times this article has been viewed
}

\section{Hongsheng Yu* \\ Yong Qu* \\ Qingjun Shang \\ Chao Yan \\ Peng Jiang \\ Xiang Wang \\ Donghai Liang \\ Tao Jiang}

Department of Oncology, Affiliated Hospital of Qingdao University,

Qingdao, People's Republic of China

*These authors contributed equally to this work
Correspondence: Hongsheng Yu Department of Oncology, Affiliated Hospital of Qingdao University, No 16 Jiangsu Road, Qingdao 266000, Shandong, People's Republic of China

Tel +86532 8291 1847

Email hongshyu@I26.com
Objective: The objective of this study was to explore the clinical effects of low-dose splenic irradiation on locally advanced non-small-cell lung cancer (NSCLC) patients.

Methods: Thirty-eight patients with stage III NSCLC were randomly divided into a control group and a combined treatment group. The control group only received chest three-dimensional conformal radiotherapy, while the combined treatment group received low-dose splenic irradiation followed by chest three-dimensional conformal radiotherapy after 6 hours. T lymphocyte subsets of the blood cells were tested before, during, and after treatment once a week. The side effects induced by radiation were observed, and a follow-up was done to observe the survival statistics.

Results: The ratio differences in $\mathrm{CD}^{+}$cells, $\mathrm{CD} 8^{+}$cells, and $\mathrm{CD} 4^{+} / \mathrm{CD} 8^{+}$before and after treatment were not statistically significant $(P>0.05)$ in both the groups. The immune indexes were also not statistically significant $(P>0.05)$ before and after radiotherapy in the combined treatment group. However, the numbers of $\mathrm{CD}^{+}$cells and $\mathrm{CD} 4^{+} / \mathrm{CD} 8^{+}$ratios before radiotherapy were higher than after radiotherapy in the control group. There were no differences in the incidence of radiation toxicities between the two groups; however, the incidence of grade III or IV radiation toxicities was lower, and the dose at which the radiation toxicities appeared was higher in the combined treatment group. The total response rate was $63.16 \%$ $(12 / 19)$ in the combined treatment group vs $42.11 \%(8 / 19)$ in the control group. The median 2 -year progression-free survival (15 months in the combined treatment group vs 10 months in the control group) was statistically significant $(P<0.05)$. The median 2 -year overall survival (17.1 months in the combined treatment group vs 15.8 months in the control group) was not statistically significant $(P>0.05)$.

Conclusion: Low-dose radiation can alleviate the radiation toxicities, improve the short-term efficacy of radiotherapy, and improve the survival of locally advanced NSCLC patients.

Keywords: non-small-cell lung cancer, low-dose splenic irradiation, clinical effects, immune function, radiation toxicities

\section{Introduction}

In terms of the biological effects of radiation on human beings, the majority of researchers support the notion of linear on threshold that all dose levels of radiation are harmful when given for many years. However, Luckey stated in the 1980s that lowdose radiation (LDR) could induce positive responses in human beings. ${ }^{1}$ The United Nations Scientific Committee on the Effects of Atomic Radiation formulated in 1986 
that the LDR refers to a dose rate that is $<0.05 \mathrm{mGy} / \mathrm{min}$ of irradiation, which is a low-linear energy transfer radiation within 0.2 Gy or a high-linear energy transfer radiation within $0.05 \mathrm{~Gy}^{2}$ Since then, the research and epidemiological surveys on the biological effects of LDR have become more common. In recent years, studies have revealed that LDR is beneficial to human beings. It can promote growth, enhance immunity, improve one's adaptive capacity, and accelerate the repair of damage. ${ }^{3-7}$ Studies have also demonstrated that LDR can improve the individuals' ability to defend against tumors and can alleviate the side effects of chemotherapy and radiotherapy. ${ }^{8-10}$ The hormesis and adaptive responses induced by LDR are now widely accepted by more and more researchers. Although its concrete mechanism needs to be explored further, researchers have known that one of the most important pathways is to activate the immune system.

As we know, the immune system plays an important role in the emergence and progress of malignant tumors. The spleen is the largest immune organ in the human body, where many lymphocytes live and many important immune reactions take place. Experiments have proven that LDR can have beneficial effects on the human immune system when the spleen receives the irradiation. ${ }^{1-13}$ LDR can boost the functions of the spleen cells. It can stimulate the secretion of interleukin- 2 and interferon- $\gamma$, activate natural killer cells and antigen-dependent cell-mediated cytotoxicity, and accelerate the proliferation of immune cells in the spleen. Therefore, we know that LDR can have positive effects on the human body by stimulating the functions of the spleen. However, all these results were obtained from laboratory studies, and only few clinical trials have been done. The clinical effects of LDR combined with radiotherapy deserve to be studied.

Lung cancer is still the leading cause of cancer death worldwide. Approximately 30\%-40\% patients with nonsmall-cell lung cancer (NSCLC) have been in stage III when they were first diagnosed. Radiotherapy combined with chemotherapy is the recommended antineoplastic protocol, but the 5-year survival rate of stage IIIA NSCLC is $15 \%-23 \%$, and that of stage IIIB NSCLC is only $6 \%-7 \% ;{ }^{14}$ obviously, it remains to be improved. Radiotherapy is an important method to improve the local control rate, and with three-dimensional conformal radiotherapy, it is possible to accurately irradiate the target area while significantly reducing the radiation dose received by the surrounding normal tissues and organs. However, the side effects of radiation, particularly the grade III or IV toxicities, still often delay treatment, which has limited the efficiency of radiotherapy. Many researchers have reported that pretreatment with LDR can induce damage resistance to the following high-dose irradiation. ${ }^{15-17}$ The special biological effects of LDR imply that it can be a complementary pathway of existing treatment. Therefore, our research group designed this study to explore whether LDR can alleviate the degree of radiation toxicities and improve the efficacy of clinical treatments by affecting the immune system in locally advanced NSCLC patients.

\section{Methods}

\section{Inclusion criteria}

The inclusion criteria of the study were as follows: presence of either pathologically or cytologically confirmed, previously untreated NSCLC, at the clinical stage of IIIA or IIIB (as defined by the NSCLC staging standards of National Comprehensive Cancer Network [NCCN] 2011) or NSCLC that had recurred after surgery; age of 18-75 years; the Eastern Cooperative Oncology Group scores of 0 or 1 ; and an expected survival time of at least 3 months. The laboratory test results criteria were as follows: white blood cell counts of at least $3.5 \times 10^{9} / \mathrm{L}$, neutrophil counts of $>1.5 \times 10^{9} / \mathrm{L}$, hemoglobin levels of at least $90 \mathrm{~g} / \mathrm{L}$, platelet counts of at least $100 \times 10^{9} / \mathrm{L}$, creatinine clearance rates of at least $50 \mathrm{~mL} / \mathrm{min}$, serum aspartate and alanine aminotransferase levels that were 2.5 times lower than the normal range, serum total bilirubin level that was 1.5 times lower than the normal range, and no presence of significant electrocardiographic abnormalities. The patients were required to follow treatment protocol and come for a follow-up visit.

\section{Exclusion criteria}

Patients were excluded from the study if they were pregnant or lactating or had a planned pregnancy. Patients who suffered from active infections or severe lung disorders that affect lung function were excluded. Patients who suffered from mental illness and could not follow the trial protocol were also excluded.

This clinical trial was approved by the Ethics Committee of the Affiliated Hospital of Qingdao University. This study was performed in accordance with the standards for human clinical trials and the principles stated in the Declaration of Helsinki (as issued in 1975 and revised in 2000). All the patients signed an informed consent form prior to enrollment. They were randomly divided into a control group (D1) and a combined treatment group (D1 + D2).

\section{Patient assessment}

Patients underwent assessment within 2 weeks prior to the treatment, including an evaluation of complete medical history, a comprehensive physical examination, thoracic and 
abdominal computed tomography (CT), magnetic resonance imaging of the head, an electrocardiogram, transbronchial lung biopsy or CT-guided needle biopsy or sputum cytology, routine blood testing, a comprehensive blood biochemical profile testing, and a whole-body bone scan with emission CT. The patients underwent physical examinations and a quality-of-life assessment and had routine blood testing performed each week (with an increased frequency of examinations if necessary).

\section{Treatment}

Patients in both the groups underwent three-dimensional conformal radiotherapy (Figure 1). They were in the supine position with their hands folded on top of their heads. A vacuum pad was used to immobilize each patient's body position and appropriately limit respiratory motion. A chest CT that can include the spleen was performed. The image data were input into the three-dimensional treatment planning system. The Venus software package (Visual Domains Ltd., Tel Aviv, Israel) was used to design the radiotherapy plan. The full set of U.S. Varian radiotherapy equipment (Varian Medical Systems, Palo Alto, CA, US) was utilized to complete the treatment, which included a linear accelerator $23 \mathrm{EX}$, a full set of carbon fiber-fixed positioning devices, an IBA radiotherapy quality control system (Ion Beam Applications S.A., LouvainLa-Neuve, Belgium), and a radiotherapy network.
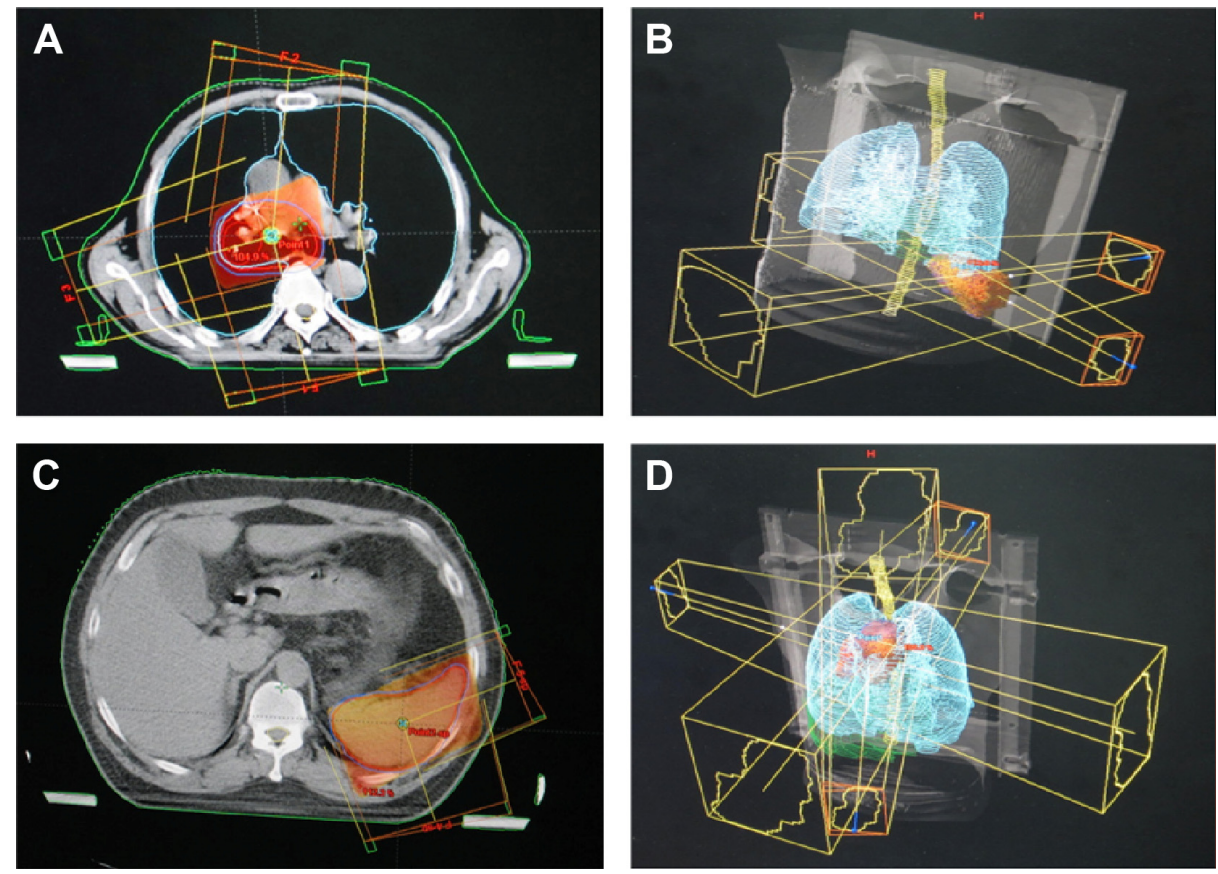

Figure I Target design of the chest and the spleen.

Notes: (A) Target design of the chest (two-dimensional). (B) Target design of the chest (three-dimensional). (C) Target design of the spleen (two-dimensional). (D) Target design of the spleen (three-dimensional). 
and two wide, with or without wedge plate. GTV2-sp was defined as the anatomical boundaries of spleen. CTV2-sp was the same as GTV2-sp. CTV2-sp was enlarged $1 \mathrm{~cm}$ to form PTV2-sp. The dose of PTV2-sp was $7.5 \mathrm{cGy} /$ fraction with two fractions per week (on Monday and Thursday) until the end of the chest three-dimensional conformal radiotherapy.

The control group (D1) only received the chest threedimensional conformal irradiation; the combined treatment group $(\mathrm{D} 1+\mathrm{D} 2)$ received low-dose splenic irradiation and chest three-dimensional conformal irradiation, that is, pretreatment low-dose splenic irradiation and then chest threedimensional conformal radiotherapy after 6 hours.

\section{Research indexes}

The peripheral blood T lymphocyte subsets, which include numbers of $\mathrm{CD}^{+}$cells and $\mathrm{CD}^{+}$cells of all patients, were detected by flow cytometry before, during, and after treatment. The white blood cells, neutrophils, hemoglobin, and platelet counts were measured by Japan Sysmex (Sysmex Corporation, Kobe, Japan) in terms of five categories of blood cells. The toxicities induced by radiation (acute radiation pneumonia, acute radiation esophagitis, acute radiation dermatitis, gastrointestinal reactions, and bone marrow suppression) were observed.

\section{Follow-up and statistics}

All patients received a chest CT scan to evaluate the shortterm efficacy of the radiotherapy 1 month after the completion of the radiotherapy. Then, a follow-up was conducted every $2-3$ months. All the statistical analyses were performed using the SPSS 19.0 biostatistical software package (IBM Corporation, Armonk, NY, USA). Only the first treatment failure was taken into account. The dependency of the shortterm efficacy (complete response, partial response, stable disease, and progressive disease) was determined according to the standard Response Evaluation Criteria in Solid Tumors (version 1.1). Progression-free survival (PFS) was defined as survival without local recurrence or distant metastases. The survival data were evaluated using the Kaplan-Meier method. The survival time was measured from the initiation of the radiotherapy until death due to any cause or the subsequent follow-up event.

\section{Results}

\section{Patient characteristics}

Thirty-eight patients in the Tumor Radiotherapy Center of our hospital (The Affiliated Hospital of Medical College, Qingdao University, People's Republic of China) with pathologically or cytologically confirmed NSCLC were enrolled in this trial from July 2011 to July 2013. The general characteristics of these patients are provided in Table 1 . The median age was 60 years in the combined treatment group vs 63 years in the control group, and the Eastern Cooperative Oncology Group score was not $>1$ in both the groups. The sample included 13 cases of squamous cell carcinoma, 17 cases of adenocarcinoma, three cases of large cell carcinoma, three cases of adenosquamous carcinoma, and two cases of undifferentiated carcinoma. There were 14 cases of stage IIIA and 24 cases of stage IIIB (including three cases of supraclavicular lymph node metastasis) among patients with previously untreated NSCLC.

\section{T lymphocyte subsets}

The ratio differences in $\mathrm{CD} 4^{+}$cells, $\mathrm{CD} 8^{+}$cells, and $\mathrm{CD} 4^{+} / \mathrm{CD}^{+}$ before and after treatment were not statistically significant $(P>0.05)$ in both the groups. The immune indexes were also not statistically significant $(P>0.05)$ before and after radiotherapy in the combined treatment group. However, in the control group, they were statistically significant $(P<0.05)$ before and after radiotherapy. The numbers of $\mathrm{CD}^{+}$cells and $\mathrm{CD}^{+} / \mathrm{CD}^{+}$ratios before radiotherapy were also higher than after radiotherapy in the control group (Figure 2).

\section{Radiation toxicities}

No treatment-related death occurred in both the groups. There were no differences in the incidence of acute radiation

Table I Patient characteristics

\begin{tabular}{|c|c|c|c|c|}
\hline \multirow[t]{2}{*}{ Characteristics } & \multicolumn{2}{|c|}{$\begin{array}{l}\text { No of patients } \\
(\mathrm{N}=38)\end{array}$} & \multicolumn{2}{|c|}{$\begin{array}{l}\text { Percentage of } \\
\text { patients (\%) }\end{array}$} \\
\hline & $D I+D 2$ & DI & DI + D2 & DI \\
\hline \multicolumn{5}{|l|}{ Sex } \\
\hline Male & 17 & 16 & 89.5 & 84.2 \\
\hline Female & 2 & 3 & 10.5 & 15.8 \\
\hline \multicolumn{5}{|l|}{ Age (years) } \\
\hline Median & 60 & 63 & & \\
\hline Range & $43-74$ & $56-72$ & & \\
\hline \multicolumn{5}{|l|}{ ECOG } \\
\hline Range & $0-1$ & $0-1$ & & \\
\hline \multicolumn{5}{|l|}{ Histology } \\
\hline Squamous cell carcinoma & 6 & 7 & 31.6 & 36.8 \\
\hline Adenocarcinoma & 8 & 9 & 42.1 & 47.4 \\
\hline Large cell carcinoma & 2 & I & 10.5 & 5.3 \\
\hline Adenosquamous carcinoma & 1 & 2 & 5.3 & 10.5 \\
\hline Undifferentiated carcinoma & 2 & 0 & 10.5 & 0 \\
\hline \multicolumn{5}{|l|}{ Stage } \\
\hline IIIA & 8 & 6 & 42.1 & 31.6 \\
\hline IIIB & 11 & 13 & 57.9 & 68.4 \\
\hline
\end{tabular}

Notes: DI + D2: combined treatment group; DI: control group. Abbreviation: ECOG, Eastern Cooperative Oncology Group. 

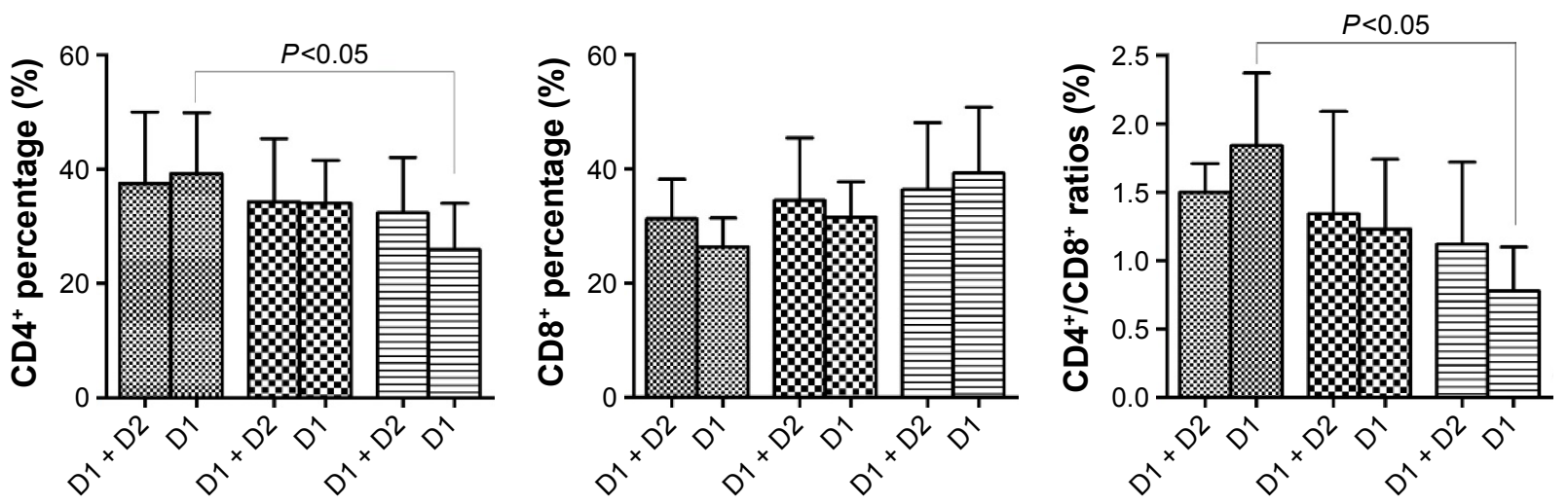

Before treatment $\mathbf{E}$ During treatment

Figure 2 Percentages of $\mathrm{CD} 4^{+}$cells and $\mathrm{CD} 8^{+}$cells and $\mathrm{CD} 4^{+} / \mathrm{CD} 8^{+}$ratios detected before, during, and after treatment.

Notes: DI + D2: combined treatment group; DI: control group.

pneumonia, acute radiation esophagitis, gastrointestinal reactions, and bone marrow suppression between the two groups. But the incidences of grade III or IV of the radiation toxicities were lower (Table 2). We chose acute radiation pneumonia, acute radiation esophagitis, and bone marrow suppression to observe the total dose at which these side effects first appeared. The result was that the dose at which the radiation toxicities appeared was higher in the combined treatment group than in the control group (Table 3).

\section{Short-term treatment efficacy}

The results of the evaluations of the short-term treatment efficacy for the 38 cases were as follows: there was no complete response, the rate of partial response was $63.16 \%$ $(12 / 19)$ in the combined treatment group vs $42.11 \%(8 / 19)$ in the control group, the rate of stable disease was $36.84 \%$ $(7 / 19)$ in the combined treatment group vs $52.63 \%(10 / 19)$ in the control group, and the rate of progressive disease was $0 \%(0 / 19)$ in the combined treatment group vs $5.26 \%(1 / 19)$ in the control group. The total response rate was $63.16 \%$

Table 2 The side effects

\begin{tabular}{|c|c|c|c|c|}
\hline Item & Grade I & Grade II & Grade III & Grade IV \\
\hline & DI (DI + D2) & DI (DI + D2) & $D I(D I+D 2)$ & $D I(D I+D 2)$ \\
\hline \multicolumn{5}{|l|}{ Acute } \\
\hline Radiation pneumonia & $3(2)$ & I (0) & $0(0)$ & $0(0)$ \\
\hline Cough & $7(6)$ & $2(2)$ & I (0) & $0(0)$ \\
\hline Radiation esophagitis & $7(5)$ & $4(3)$ & I (0) & $0(0)$ \\
\hline Radiation dermatitis & $6(3)$ & I (0) & $0(0)$ & $0(0)$ \\
\hline Nausea & $6(3)$ & $3(1)$ & $2(0)$ & $0(0)$ \\
\hline Vomiting & $5(6)$ & $2(0)$ & $0(0)$ & $0(0)$ \\
\hline Anorexia & $9(7)$ & $5(2)$ & I (0) & $0(0)$ \\
\hline Fatigue & $8(6)$ & $4(2)$ & $I(I)$ & $0(0)$ \\
\hline Neutropenia & $6(6)$ & $8(7)$ & $3(1)$ & $2(0)$ \\
\hline Thrombocytopenia & $4(4)$ & $2(1)$ & I (0) & $0(0)$ \\
\hline Anemia & $7(5)$ & $2(0)$ & $0(0)$ & $0(0)$ \\
\hline ALT & I (0) & $0(0)$ & $0(0)$ & $0(0)$ \\
\hline AST & I (0) & $0(0)$ & $0(0)$ & $0(0)$ \\
\hline $\mathrm{Cr}$ & $0(0)$ & $0(0)$ & $0(0)$ & $0(0)$ \\
\hline BIL & $0(0)$ & $0(0)$ & $0(0)$ & $0(0)$ \\
\hline \multicolumn{5}{|l|}{ Late } \\
\hline Lung & $5(2)$ & $2(0)$ & $0(0)$ & $0(0)$ \\
\hline Esophagus & I (0) & $0(0)$ & $0(0)$ & $0(0)$ \\
\hline Skin & $2(I)$ & $0(0)$ & $0(0)$ & $0(0)$ \\
\hline
\end{tabular}

Notes: DI + D2: combined treatment group; DI: control group.

Abbreviations: ALT, alanine aminotransferase; AST, aspartate aminotransferase; $\mathrm{Cr}$, serum creatinine; BIL, bilirubin. 
Table 3 The dose at which the radiation toxicities appear

\begin{tabular}{|c|c|c|c|c|}
\hline \multirow[t]{2}{*}{ Complications } & \multicolumn{2}{|c|}{ DI + D2 } & \multicolumn{2}{|l|}{ DI } \\
\hline & $\mathbf{N}$ & Average dose & $\mathbf{N}$ & Average dose \\
\hline Acute radiation pneumonia & 2 & $42.23 \pm 3.68$ & 4 & $38.23 \pm 3.68$ \\
\hline Acute radiation esophagitis & 8 & $35.3 I \pm 4.17$ & 12 & $28.53 \pm 3.58$ \\
\hline Bone marrow suppression (above grade III) & I & $34.23 \pm 3.42$ & 6 & $25.67 \pm 2.89$ \\
\hline
\end{tabular}

Notes: DI + D2: combined treatment group; DI: control group. Data is presented as mean \pm standard deviation.

$(12 / 19)$ in the combined treatment group vs $42.11 \%(8 / 19)$ in the control group.

\section{Survival}

The median follow-up period was 13.4 months in the combined treatment group vs 11.5 months in the control group. The median 2-year PFS was 15 months in the combined treatment group vs 10 months in the control group $(P=0.254)$ (Figure 3A). The median 2-year overall survival was 17.1 months in the combined treatment group vs 15.8 months in the control group ( $P=0.952)$ (Figure 3B). The reasons for treatment failure were as follows: one case in the combined treatment group and two cases in the control group had a local recurrence, two cases in the combined treatment group and four cases (two cases of brain metastasis, one case of liver metastasis, one case of bone metastasis) in the control group had distant metastases, and one case in the control group died of other diseases.

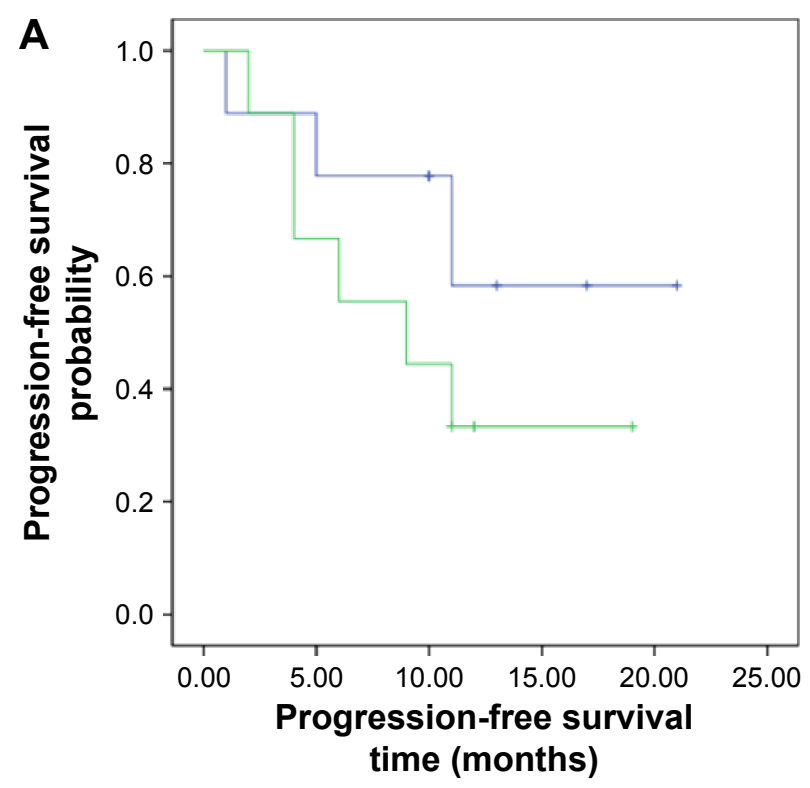

\section{Discussion}

In our clinical trial, we evaluated the clinical effects of LDR in terms of the immune indexes ( $\mathrm{T}$ lymphocyte subsets), the radiation toxicities, the short-term efficacy, and the 2-year survival data. Positive data were obtained for all of these. In the following section, we explain these findings and their concrete mechanisms.

Radiotherapy is one of the most important methods used to treat tumors, but it can also induce different degrees of immune damages. The suppression of the immune system is the main reason for tumor metastasis and treatment failure. Hashimoto et al have reported that low-dose total-body irradiation significantly decreased the incidence of lung and lymph node metastases, whereas the same dose of local irradiation had no effect on the incidence of metastasis, and the tumor tissue-infiltrating lymphocytes were significantly increased after low-dose total-body irradiation. ${ }^{18}$ These results implied that LDR can inhibit the metastasis of malignant tumors by

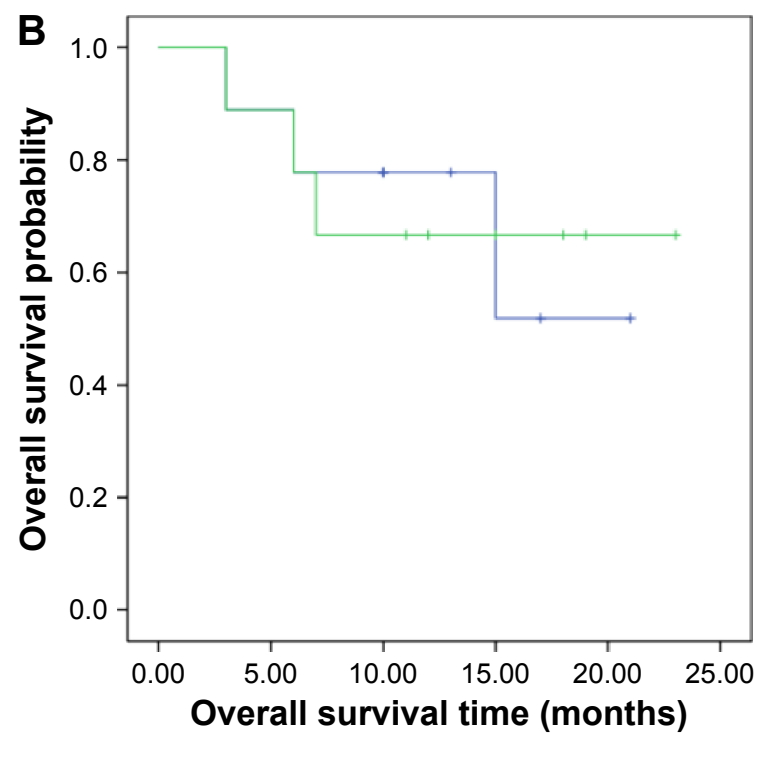

$$
\neg \text { Combined treatment group } \neg \text { Control group }
$$

Figure 3 The survival time.

Notes: (A) The median 2-year progression-free survival was 15 months in the combined treatment group vs 10 months in the control group ( $P=0.254)$. (B) The median 2-year overall survival was 17.1 months in the combined treatment group vs 15.8 months in the control group $(P=0.952)$. 
stimulating the immune system but not by the direct effect of rays. The cell immunity mediated by the $\mathrm{T}$ lymphocytes is the main defense against tumors. T lymphocytes can be classified into different subsets, two of which are the CD4 ${ }^{+} \mathrm{T}$ lymphocytes and CD8 ${ }^{+} \mathrm{T}$ lymphocytes. The number and ratio changes of $\mathrm{CD}^{+}$cells and $\mathrm{CD}^{+}$cells can reflect changes in the immune system's function. A reduction in $\mathrm{CD}^{+}$cells can cause the immune escape of tumor cells, and an increase in $\mathrm{CD}^{+}$cells can cause immune damage. LDR can boost the proliferation of lymphocytes, and at the same dose level, the proliferation of $\mathrm{CD}^{+}$lymphocytes is more active than $\mathrm{CD} 8^{+}$lymphocytes. ${ }^{19}$ The results of evaluation of the peripheral blood $\mathrm{T}$ lymphocyte subsets in our clinic trial demonstrated that LDR has positive effects on the immune functions of patients with lung cancer. In the control group, the numbers of $\mathrm{CD}^{+}$lymphocytes and the $\mathrm{CD} 4^{+} / \mathrm{CD}^{+}$ratios were reduced, but this was not observed in the combined treatment group. This means that LDR can reduce the suppression of the immune system caused by radiotherapy, and thus reduce the incidence of distant metastasis and improve the patients' survival rate.

Compared with the control group, the combined treatment group in our clinical trial had a lower incidence of grade III or IV radiation toxicities, and the dose at which the radiation toxicities appeared was higher. These toxicities included acute radiation pneumonia, acute radiation esophagitis, gastrointestinal reactions, and bone marrow suppression. It is worth noting that two patients in the combined treatment group received a total dose of 74 Gy without developing grade III or IV radiation toxicities. These results mean that LDR can improve the tolerance of the organ at risk. It was also known that the radiation dose depends on the tolerance dose of the organ involved in the planning target volume and the lethal dose of the tumor, and the tolerance dose of the organ involved in the planning target volume is the most important. Improving the tolerance of the organ at risk provides us with an opportunity to use higher doses to treat tumors and ensure that the treatment process goes smoothly, thus giving us more opportunities to control tumors. The concrete mechanisms behind this are probably as follows. Experiments have proven that LDR can induce the generation of colony-forming unit - granulocyte-macrophage and burstforming unit - erythroid in bone marrow and boost the secretion of granulocyte-macrophage colony-stimulating factor. ${ }^{20}$ These were caused by the improved transcriptional level in spleen cells. Meanwhile, giving LDR before treatment can improve the normal organ's resistance to high-dose radiation. LDR can activate enzymes that assist to produce DNA and protein, thus improving the repairing ability of normal cells. ${ }^{21}$ LDR can also improve the superoxide dismutase activity, which can oxidize free radicals and transform them into $\mathrm{H}_{2} \mathrm{O}$; therefore, it can alleviate the damage caused by free radicals produced by high-dose X-rays. ${ }^{22}$

Distant metastasis and local recurrence are the main reasons for treatment failure. The resistance of tumor hypoxic cells to radiotherapy plays an important role in the distant metastasis and local recurrence after radiotherapy. Experiments have revealed that HIF-1 is one of the hypoxic biomarkers, and LDR can decrease the expression of HIF-1 to change the hypoxic status of tumor cells. ${ }^{23}$ The change of hypoxic status in tumor cells will improve the tumor's sensitivity to radiotherapy, thus improving the efficiency of the radiotherapy. Therefore, the total response rate was better in the combined treatment group than in the control group in our clinical trial. On the other hand, LDR can reduce the expression of matrix metalloproteinases, which can break down the extracellular matrix to inhibit the infiltration and metastasis of tumors. ${ }^{24}$ Therefore, we had fewer cases of progression in the combined treatment group. Although the number of cases enrolled in our clinical trial was insufficient and the follow-up time was short, we still obtained better survival data, and the results also support the abovementioned views. We can see that the 2-year PFS in the combined treatment group was significantly longer than in the control group.

\section{Conclusion}

The hormesis and the adaptive response induced by LDR have been approved by researchers around the world. Although the cases in our clinical research were insufficient, and more cases and a longer follow-up time are needed, our clinical data still provide strong support for the clinical application of LDR. LDR-mediated radioadaptive response is crucial for developing potential therapeutic approaches to improve normal tissue protection in radiotherapy. LDR represents a new form of radiotherapy for treating malignant tumors. It promotes radiotherapy from a local treatment approach to a whole-body treatment approach by activating the immune system. We believe that this will be an important part of comprehensive tumor treatment in the future.

\section{Acknowledgment}

This work was supported by the Natural Science Foundation of Shandong Province, China (No ZR2015HM025) and Science and Technology Development Program of Shandong Province, China (No 2011YD18003). 


\section{Disclosure}

The authors report no conflicts of interest in this work.

\section{References}

1. Luckey T. Physiological benefits from low levels of ionizing radiation. Health Phys. 1982;43(6):771-789.

2. Hei TK, Zhou H, Chai Y, Ponnaiya B, Ivanov VN. Radiation induced non-targeted response: mechanism and potential clinical implications. Curr Mol Pharm. 2011;4(2):96-105.

3. Mothersill C, Seymour C. Implications for human and environmental health of low doses of ionising radiation. $J$ Environ Radioact. 2014; 133:5-9.

4. Serebrianyi AM, Aleshchenko AV, Antoshchina MM, et al. [The radiosensitivity change of human blood lymphocytes in different mitotic cycles after a low dose irradiation]. Radiats Biol Radioecol. 2008;48(6):713-720. Italian.

5. Sakai K. [Biological responses to low dose radiation - hormesis and adaptive responses]. Yakugaku Zasshi. 2006;126(10):827-831. Japanese.

6. Luo-Owen X, Pecaut MJ, Rizvi A, Gridley DS. Low-dose total-body $\gamma$ irradiation modulates immune response to acute proton radiation. Radiat Res. 2012;177(3):251-264.

7. Bogdandi EN, Balogh A, Felgyinszki N, et al. Effects of low-dose radiation on the immune system of mice after total-body irradiation. Radiat Res. 2010;174(4):480-489.

8. Li T, Lang JY, Wang J, Deng B, Xie Z, Wang J. Effects of low dose fractionated irradiation on spleen to improve the anti-irradiation function in esophageal carcinoma. Cancer Res Clin. 2003;15:234-236.

9. Liu Q, Yu HS, Xue HW, Song AQ, Shen FZ, Liang J. Effects of low dose radiation combined with cyclophosphamide on tumor cell apoptosis, cell cycle and proliferation of bone marrow in tumor-bearing mice. Med J Qilu. 2008;23(1):3-5.

10. Yu H, Sun W, Liu N. The effect of low-dose total body irradiation on tumor-inhibition and signal transduction in tumor tissues of mice bearing S180 sarcoma. Chin Ger J Clin Oncol. 2011;10:602-605.

11. Kojima S. [Induction of glutathione and activation of immune functions by low-dose, whole-body irradiation with gamma-rays]. Yakugaku Zasshi. 2006;126(10):849-857. Japanese.
12. Yu H, Liu N, Ju B. Low dose radiation induced adaptive response of apoptosis in mouse spleen cells. Chin Ger J Clin Oncol. 2010;9: 235-238.

13. Li T, Lang JY, Lu Y. Effect of low dose radiation on expression of apoptosis-related gene products. Chin Clin Oncol. 2004;9:371-373.

14. Liu J, Yu H, Shang Q, Yan C, Jiang P, Wang X. The effects of low-dose splenic irradiation and radiotherapy on immune system of patients with locally advanced non-small cell lung cancer. Chin Ger JClin Oncol. 2013; 12:51-55.

15. Takahashi A, Ohnishi K, Asakawa I, et al. Radiation response of apoptosis in C57BL/6N mouse spleen after whole-body irradiation. Int J Radiat Biol. 2001;77(9):939-945.

16. Rigaud O, Moustacchi E. Radioadaptation for gene mutation and the possible molecular mechanisms of the adaptive response. Mutat Res. 1996;358(2):127-134.

17. Ikushima T, Aritomi H, Morisita J. Radioadaptive response: efficient repair of radiation-induced DNA damage in adapted cells. Mutat Res. 1996;358(2):193-198.

18. Hashimoto S, Shirato H, Hosokawa M, et al. The suppression of metastases and the change in host immune response after low-dose total-body irradiation in tumor-bearing rats. Radiat Res. 1999;151(6):717-724.

19. Qiong W, Jun Z, Mingli W. Detection and analysis of T cell subsets in peripheral blood of aged mice infected with Human cytomegalovirus. Acta Univ Med Anhui. 2010;3:008.

20. Wang G, Tan Y, Zhang F, Li W, Yang L, Ma L. [The study of low dose radiation inducing hormesis effect on hematopoietic system]. Zhonghua Xue Ye Xue Za Zhi. 2001;22(5):232-234. Chinese.

21. Suzuki K, Kodama S, Watanabe M. Suppressive effect of low-dose preirradiation on genetic instability induced by $\mathrm{X}$ rays in normal human embryonic cells. Radiat Res. 1998;150(6):656-662.

22. Yu HS, Song AQ, Lu YD, Qiu WS, Shen FZ. Effects of low-dose radiation on tumor growth, erythrocyte immune function and SOD activity in tumor-bearing mice. Chin Med J (Engl). 2004;117(7):1036-1039.

23. Li F, Yu H. The effect of low-dose radiation on HIF-1 $\alpha$ and P53 in tumor tissue of S180-sarcoma-bearing mice. Med J Qilu. 2009;24: 189-191.

24. Zhang Y, Yu H. Effect of low dose radiation on tumor expression of MMP-2 and TIMP-2 in S180 sarcoma-bearing mice. Med J Qilu. 2010; $25: 214-216$
OncoTargets and Therapy

\section{Publish your work in this journal}

OncoTargets and Therapy is an international, peer-reviewed, open access journal focusing on the pathological basis of all cancers, potential targets for therapy and treatment protocols employed to improve the management of cancer patients. The journal also focuses on the impact of management programs and new therapeutic agents and protocols on

\section{Dovepress}

patient perspectives such as quality of life, adherence and satisfaction The manuscript management system is completely online and includes a very quick and fair peer-review system, which is all easy to use. Visit http://www.dovepress.com/testimonials.php to read real quotes from published authors. 